\title{
Eine Poetologie nach Krieg und Katastrophe: Erich Arendts "Der Albatros" (1951) und Ingeborg Bachmanns „Mein Vogel" (1956)
}

\section{A Poetology After War and Catastrophy. Erich Arendt's "Der Albatros" ["The Albatros"] (1951) and Ingeborg Bachmann's "Mein Vogel" ["My Bird"] (1956)}

Jana Hrdličková

\begin{abstract}
The poems "Der Albatros" by Ernst Arendt and "Mein Vogel" by Ingeborg Bachmann are well known parts of the oeuvre of the two authors. Most interpretations concentrate on their autobiographical or poetological elements, without taking much notice of both texts reflecting the events of the time in a clearly involved manner; this is achieved despite their hermetic characteristics, which are indeed helpful for the authors to devise a poetology that tries to do justice to the facts of World War II and the Shoa.

The aim of this article is to give evidence of these aspects by "close reading" of the two texts and to show, by examining a few selected passages, what the poems have in common and where they differ in presenting contemporary events.
\end{abstract}

\section{Keywords}

poetology after World War II; hermetic poetry; Austrian and German literature of the $20^{\text {th }}$ century 
Erich Arendt (1903-1984) und Ingeborg Bachmann (1926-1973) werden kaum in einem Atemzug genannt. Zwar stammen sie beide aus der Provinz (doch einer wie unterschiedlichen: der preußischen und der österreichischen) und werden beide der Hermetik zugerechnet, doch das ändert nichts an der Tatsache, dass sie unterschiedlichen Generationen angehören und einen sehr unterschiedlichen biographischen Hintergrund haben. Denn Arendt wuchs in ziemlich bescheidenen Verhältnissen auf und litt sehr darunter, während Bachmanns Familie ein eigenes Haus bewohnte und sogar über eine Haushaltshilfe mitten im Krieg verfügte - was sich aber nicht minder traumatisch auf die künftige Dichterin auswirken sollte. Bachmanns Vater trat nämlich früh der NSDAP bei und beteiligte sich als Oberleutnant aktiv an den Kämpfen gegen die Sowjetunion im Zweiten Weltkrieg, wohingegen Arendt als Linker vor Hitler fliehen musste und sein Exil v.a. in Kolumbien verbrachte; um schließlich, 1950, in der DDR anzukommen, doch äußerst unzufrieden mit deren Regime.

Trotz dieser lebensweltlichen Differenzen gibt es im Werk von beiden Autoren zwei Gedichte, die von ihnen wie von der Forschung jeweils als wichtig eingestuft werden und darüber hinaus miteinander zu korrespondieren scheinen: Erich Arendts Der Albatros und Ingeborg Bachmanns Mein Vogel. Ihr Vergleich, der die beiden Gedichte hermeneutisch aufzuschließen versucht und mithilfe „close reading“ einiger zentraler Stellen bisher Unbemerktes an den Tag legen soll, zeigt einen Aspekt, der in der Arendtwie Bachmann-Philologie bisher kaum herausgearbeitet wurde: den engen Bezug beider Gedichte zu ihrer Zeit, in gesellschaftskritischer und politischer Hinsicht. Die folgende Abhandlung soll diesem Desiderat detailliert nachgehen.

Wie man in der Heraldik stilisierte Tiere verwendet, um auf den bestimmten Wappeninhaber und seine Spezifika bzw. Devisen hinzuweisen, so begegnen wir im Arendtschen Gedicht „Der Albatros“ (1951) und im Bachmannschen Gedicht „Mein Vogel“ (1956) jeweils einer Vogelart, die für den Autor/die Autorin stehend und redend sein soll: dem Sturmvogel Albatros (Arendt) und dem Nachtvogel Eule (Bachmann). Beide sind autobiographisch begründet (Arendt identifizierte sein unruhiges, gefahrenvolles Leben mit diesem Vogel, dem Begleiter seiner Irrfahrten, vor allem seiner Flucht aus Europa; schon in der Schulzeit wurde Bachmann L'hibou, die Eule, genannt ${ }^{1}$ ) und beide werden im Text possessiv dem lyrischen Ich als „mein“ zugeeignet; und zwar im Finale des Gedichts von Arendt sowie im Titel und an etlichen Signalstellen des Gedichts von Bachmann. Für Arendt ist zudem der Vogel so wichtig, dass er nicht nur das eine Gedicht (Emmerich und Ullrich zufolge 1941 bei der schicksalshaften Überfahrt nach Kolumbien entstanden ${ }^{2}$ ) nach ihm benennt, sondern die ganze erste Gedichtsammlung (die erste Buchveröffentlichung Arendts überhaupt) mit Gedichten aus den Jahren 1931-1950: Trug doch die Nacht den Albatros.

In ihrem Rahmen erscheint das Gedicht „Der Albatros“ an elfter Stelle des ersten Abteils, der mit dem Sonett „Ulysses' weite Fahrt“ ansetzt und damit eine weitere emotionale Bezugsgröße Arendts vorstellt, Homers Odysseus: auch ihn als Stütze, Identifikation

1 HÖLLER 2001: 22.

2 EMMERICH 1984: 37, ULLRICH 1969. 
und Inspiration. Beide Gedichte sind gereimt und von einem regelmäßigen Strophenbau. Jede der insgesamt acht Strophen des Gedichts „Der Albatros“ zählt sieben Zeilen (allein die fünfte Strophe verfügt über nur sechs Zeilen), wobei die letzten zwei Zeilen jeder Strophe graphisch und rhythmisch hervorgehoben werden und eine Art Conclusio bilden mit dem besonders exponierten, reimtragenden Schlusswort und Schlüsselwort „Albatros“. Hier der Wortlaut des Gedichts von Arendt:

\section{„Der Albatros}

Sonnen sanken um mein Schiff und stiegen:

Wochen stiller großer Wiederkehr!

Wolkenwände sah ich ferne liegen, und sie sanken lautlos in das Meer.

Immer aber hört ichs oben fliegen:

Über mir, der weiten Fahrt Genoss, seine Schwingen schlug ein Albatros.

Lag im weißen Glanz der Meeresspiegel um mein Schiff, so senkte sich sein Flug. Seine schmalen weitgespannten Flügel glitten wassernah um Heck und Bug, segelleicht, als ob das Licht ihn trug.

Wenn die tiefe Stille ihn verdroß,

flog mit Zornesschrei der Albatros.

Mittags schwand in leeren Himmelshellen er empor; steil über grüner See.

Plötzlich löste sich ein Stein im schnellen Schlag und streifte dicht das Schiff in Lee, und ein Schnabelhieb drang in die Wellen.

Während Wasser ihm vom Leib noch floß, floh mit blutigem Fang der Albatros.

Erst im Sturmwind, der mit weißem Biß in die Tiefen fuhr, daß Nacht und Meer aufschäumten, kam er wieder, als mein Segel riß, Blitze lachten, schwarze Wogen bäumten, als die Meergruft auf mein Schiff sich schmiß:

Dunkler Pfeil, der durch den Himmel schoß, schwang durch Sturm und Licht der Albatros.

Und sein Schrei brach aus dem Herz des Sturmes hell und heiß, unfaßbar und inständig, 
von Empörung heiß, von Zorn lebendig:

Wilde Kraft des auferstandenen Sturmes.

Meiner Seele tapferster Genoss,

rief er ihren Zorn, der Albatros.

In den Abenden der heißen Meere, wenn der Purpurhimmel jäh verging, und des Dunkels schwarze Tropenschwere undurchdringbar vor den Augen hing, flog sein Schatten hörbar überm Meere.

Wenn die Nacht die Sternensicht verschloß, trug die Nacht doch stets den Albatros.

Eines Tages um die Mittagswende stieg aus ewigem Glanz das harte Riff. Palmen wuchsen aus dem Lichtgelände und die Bai umrauschten grüne Wände, in die Bucht lief einsam ein mein Schiff.

Fern am Horizont, der nackt und groß,

flog für immer fort der Albatros.

Nur in Träumen noch am Strand nach Jahren, zog er wie ums Schiff im stillen Flug. Und - da Not und Knechtschaft unerträglich waren, daß das Herz den Haß nicht mehr ertrug, stieg sein Schrei, dem großen Zorn entfahren:

Wilder Ruf, der durch den Himmel schoß, über mir, wie einst - mein Albatros!“ (ARENDT 2003: 56-57)

Schon eine flüchtige Lektüre des Gedichts zeigt, dass sein zentrales Anliegen nicht einem Porträt des Vogels dient, wie es beispielsweise Baudelaires Gedicht „Albatros“ (BAUDELAIRE 1979: 31) leistet, sondern um die Bestimmung der Beziehung des lyrischen Ich zu dem Vogel Albatros kreist. In Zeile 7 der ersten Strophe wird er als „ein Albatros" eingeführt, doch schon Zeile 5 derselben Strophe deutet die immer wieder erfolgte akustische Wahrnehmung seines Flugs an als ein unbestimmtes, spannungsgeladenes, verheißungsvolles „es“ („Immer aber hört ichs oben fliegen“). Und noch bevor wir überhaupt erfahren, dass dieses „es“ einem Vogel zugehört und wir ihn durch unser inneres Auge erblickt haben, erklärt die sechste Zeile ihn gar zu „der weiten Fahrt Genoss[en]" des Ich, was offenbar auch eine Vorbildfunktion mit einschließt (denn er ist über dem lyrischen Ich positioniert).

Die Strophen II bis VII münden dann immer von neuem in den Nominativ „der Albatros“, mit Ausnahme der Strophe VI, in welcher „die Nacht“ den Nominativ bildet; jene Nacht, die „doch stets den Albatros [trug]“, was, wie bereits erwähnt, dem ganzen 
Gedichtband den Namen gab. Zwei Substantive werden somit im Rahmen des ganzen Gedichts semantisch besonders beschwert: die (auch geschichtlich oder gar apokalyptisch zu verstehende) Nacht und der Albatros. Wobei bis zur Strophe V der Vogel eine betont dynamische, akustisch wie visuell herausragende Komponente des Gedichts verkörpert: er schlägt „seine Schwingen“ (Zeile 7), fliegt mit „Zornesschrei“ durch die Stille (Zeile 14), flieht mit „blutige[m] Fang“ hinweg (Zeile 21), wird sogar „[d]unkler Pfeil, der durch den Himmel“ schießt (Zeile 27) und „durch Sturm und Licht“ schwingt (Zeile 28), ruft schließlich stellvertretend den „Zorn“ der Seele des lyrischen Ich als ihr „tapferster Genoss" hervor (Zeile 33 und 34). Dadurch wird der Höhepunkt des Gedichts erreicht, danach trägt nur noch die Nacht, und zwar eine finstere Nacht (die „die Sternensicht verschloß“; Zeile 40), den Albatros, als Objekt: immer wieder, „stets“ (Zeile 41); bis aber der Albatros, als Subjekt wieder, „für immer“ fortfliegt (Zeile 48) und für das lyrische Ich nur noch „in Träumen“ da ist (Zeile 49). Paradoxerweise wird er erst dadurch dem lyrischen Ich eigen als „mein Albatros“.

Demgegenüber beginnt das Bachmannsche Gedicht schon mit der Tatsache - oder wenigstens der Annahme - des Besitzes des Vogels (wie sein Titel „Mein Vogel“ nahelegt), dessen nähere Bestimmung als Eule, hier parallel zum Gedicht „Der Albatros“, erst das Ende der ersten Strophe leistet. Hier der Wortlaut des Gedichts von Bachmann:

„Mein Vogel

Was auch geschieht: die verheerte Welt

Sinkt in die Dämmrung zurück,

einen Schlaftrunk halten ihr die Wälder bereit,

und vom Turm, den der Wächter verließ,

blicken ruhig und stet die Augen der Eule herab.

Was auch geschieht: du weißt deine Zeit,

mein Vogel, nimmst deinen Schleier

und fliegst durch den Nebel zu mir.

Wir äugen im Dunstkreis, den das Gelichter bewohnt.

Du folgst meinem Wink, stößt hinaus

Und wirbelst Gefieder und Fell -

Mein eisgrauer Schultergenoß, meine Waffe,

mit jener Feder besteckt, meiner einziger Waffe!

Mein einziger Schmuck: Schleier und Feder von dir.

Wenn auch im Nadeltanz unterm Baum

die Haut mir brennt

und der hüfthohe Strauch

mich mit würzigen Blättern versucht, 
wenn meine Locke züngelt,

sich wiegt und nach Feuchte verzehrt,

stürzt mir der Sterne Schutt

doch genau auf das Haar.

Wenn ich vom Rauch behelmt

wieder weiß, was geschieht,

mein Vogel, mein Beistand des Nachts,

wenn ich befeuert bin in der Nacht,

knistert's im dunklen Bestand,

und ich schlage den Funken aus mir.

Wenn ich befeuert bleib wie ich bin

und vom Feuer geliebt,

bis das Harz aus den Stämmen tritt,

auf die Wunden träufelt und warm

die Erde verspinnt,

(und wenn du mein Herz auch ausraubst des Nachts,

mein Vogel auf Glauben und mein Vogel auf Treu!)

rückt jene Warte ins Licht,

die du, besänftigt,

in herrlicher Ruhe erfliegst -

was auch geschieht.“ (BACHMANN 1982 I: 96-97)

Auch dieses Gedicht bezieht sich, und im Vergleich mit dem Arendtschen Text viel eindeutiger und direkter, zu einer äußerst negativ wahrgenommenen zeitgenössischen Wirklichkeit, denn es macht „die verheerte Welt“ bereits in der ersten Zeile zu seinem eigentlichen Ausgangspunkt; wie das Gedicht von Arendt ebenfalls in seiner ersten Zeile von „Sonnen“, die „sanken“ (und gleich, dramatisch, wieder „stiegen“) spricht, was auf immer wieder erfolgte Untergänge hindeuten kann, im Natur- sowie im geschichtlichen Zusammenhang. Der Letztere würde zudem den Gedichtbandtitel, der Zeile 41 des vorliegenden Arendtschen Gedichts entnommen (ohne die Zeitbestimmung „stets“ jedoch, was wichtig ist), rechtfertigen, und mit „Not und Knechtschaft“ der letzten Strophe korrelieren, deren Intensität so groß sein musste, „daß das [menschliche, J.H.] Herz den Haß [als Reaktion darauf, J.H.] nicht mehr ertrug“ (Zeile 51-52). In diesem Augenblick soll der gefeierte, aufgerufene, von früher bekannte Vogel mit seinem „Schrei, dem großen Zorn entfahren“ (Zeile 53), einschreiten, stellvertretend für das lyrische Ich und seinen „[w]ilde[n] Ruf“ (Zeile 53-54) nach Gerechtigkeit; aber nur in Gedanken des Ich, nicht in der Realität.

Eine solche Lesart würde den Titel des ersten Abteils der Sammlung Trug doch die Nacht den Albatros rechtfertigen, in welchem das Gedicht scheinbar unlogisch aufzufinden ist: „Das Mittelmeer“. ${ }^{3}$ Dann würden sich „Not und Knechtschaft“ auch, wenn nicht

3 Am Mittelmeer findet man nämlich sehr selten Albatrosse, wenn auch einige Schiffe dieses Namens. 
sogar vorwiegend, auf die gegenwärtige Situation des lyrischen Ich beziehen (und nicht nur auf die vergangene und bestandene „Emigrations-Odyssee des Dichters“4), jenes Ich, das „nach Jahren“ (Zeile 49) an einen offenbar mittelmeerischen Strand kommt und in seiner hoffnungslosen Situation sich daran erinnert, wie ihm der Vogel einst, als die „Sonnen sanken“ (Zeile 1), „im stillen Flug“ „ums Schiff“ (Zeile 50), das Symbol seiner Lebensfahrt, folgte. Sein Zornesschrei von damals soll auch den Zorn des lyrischen Ich über seine Gegenwart (in der DDR und über sie hinaus) versinnbildlichen, lässt den Vogel und das lyrische Ich schließlich durch das Possessivpronomen verbunden sein. Dadurch wird die Identität und Integrität des lyrischen Ich wiederhergestellt.

Auch wenn die Regime der DDR (Arendt) und Österreichs/der BRD (Bachmann) kaum vergleichbar sind, verlaufen interessanterweise „Not und Knechtschaft“ Arendts, wie sehr auch vom kommunistischen Glaubensbekenntnis des Autors ,angereichert" und zum Expressionismus tendierend, und „die verheerte Welt“ Bachmanns recht parallel, vor allem was den Bezugspunkt zu der schriftstellerischen Gegenwart der beiden Dichter angeht. Der Ausdruck „verheert“ (im Sinne „verwüstet“, „zertrümmert“; das Verb „verheeren“ meint eigentlich „mit einem Heer überziehen“) in Hinsicht auf die Nachkriegswirklichkeit erinnert an die berühmte, schwer verifizierbare Erinnerung Bachmanns, im Interview vom Dezember 1971 festgehalten:

„Es hat einen bestimmten Moment gegeben, der hat meine Kindheit zertrümmert. Der Einmarsch von Hitlers Truppen in Klagenfurt. Es war etwas so Entsetzliches, daß mit diesem Tag meine Erinnerung anfängt: durch einen zu frühen Schmerz, wie ich ihn in dieser Stärke vielleicht später überhaupt nie mehr hatte. [...] Ein ganzes Heer [beide Hervorhebungen J.H.] kam da in unser stilles, friedliches Kärnten...“ (BACHMANN 1983: 111)

Was das „Heer“ Hitlers an Verheerendem angerichtet hat, die Verheerung Europas, sollte allerdingst lange tabuisiert werden; nicht nur, um es ungeschehen oder vergessen zu machen, sondern auch, weil dafür zuerst einmal keine Worte zur Verfügug standen, weil das Thema für viele zu schmerzhaft und zu ,groß‘ war. Berühmt ist die Umschreibung Celans für das Jüngstvergangene: „das, was geschah“. ${ }^{5}$ Wenn man sich nun vergegenwärtigt, dass für Bachmann der Krieg, wie beispielsweise dem Gedicht „Alle Tage“ aus der ersten Sammlung, Die gestundete Zeit von 1953, entnommen, „alltäglich“ geworden ist (also nicht beendet wurde, sondern in Richtung Zukunft „fortgesetzt“; BACHMANN 1982 I: 46), wird die beschwörende Wendung „W/was auch geschieht“ aus ihrem Gedicht „Mein Vogel“, gerade an die Adresse Celans, einleuchtend und verständlich. Wir

4 So ULLRICH 1969: „Das Gedicht ist 1941 geschrieben, es formuliert in einem grandiosen Symbol die Emigrations-Odyssee des Dichters."

5 Typischerweise im Gedicht „Was geschah?“ aus der Sammlung Die Niemandsrose (1963) als Frage formuliert. Aber auch schon in der Ansprache anlässlich der Entgegennahme des Literaturpreises der Freien Hansestadt Bremen (1958) präsent: „Sie, die Sprache, blieb unverloren, ja, trotz allem. Aber sie musste nun hindurchgehen durch ihre eigenen Antwortlosigkeiten, hindurchgehen durch furchtbares Verstummen, hindurchgehen durch die tausend Finsternisse todbringender Rede. Sie ging hindurch und gab keine Worte her für das, was geschah; aber sie ging durch dieses Geschehen. Ging hindurch und durfte wieder zutage treten, ,angereichert" von all dem.“ (CELAN 1968: 128) 
befinden uns, und das wurde in der Bachmann-Forschung, die sich gerne dieses poetologischen Gedichts annimmt ${ }^{6}$ bisher nicht hervorgehoben, auch bei diesem Text mitten im Krieg; denn vor allem er, der Krieg, ist es, der im Hintergrund dieses Gedichts präsentisch, schleichend "geschieht“.7

Nicht nur okkupiert somit der Zweite Weltkrieg, den die Autorin als Jugendliche auf eigene Haut zu spüren bekommen musste und den sie schon damals mutig verabscheute, ${ }^{8}$ die Gegenwart und die Zukunftsaussichten, er dehnt sich zugleich in Richtung Vergangenheit aus, so dass der höchst allgemeine Krieg sich schließlich wie „[e]in roter Faden durch das Werk Ingeborg Bachmanns" (HÖLLER 2008: 124) zieht. Denn Bachmann macht uns klar und wahrnehmbar, „dass schon in den großen früheren Zeitund Erinnerungsromanen des Jahrhunderts der Krieg das Gegenüber und die entscheidende Herausforderung des Schreibens darstellte." (HÖLLER 2008: 124) Bereits beispielsweise in Marcel Prousts À la recherche du temps perdu, dieser allegorischen Suche nach der Wahrheit, von Bachmann 1958 im Bayerischen Rundfunk München besprochen, sieht die Autorin, „dass in dieser Zeit, außer dem Krieg, alles außer Kraft gesetzt ist. Aber es ist nicht der Krieg, der geschieht, wo die Schüsse fallen, oder der abgemalt werden könnte auf einem Schlachtenbild, sondern seine Spiegelung, die wirklicher ist: sein Eindringen in die Sprache aller [...]." (BACHMANN 1982 IV: 168) In ihr „geschieht“ der Krieg, in ihr muss er abgewehrt werden.

Im Gedicht „Mein Vogel“ wird zuerst sein Resultat, „die verheerte Welt“, durch die „Dämmrung“ in der Natur unsichtbar gemacht, quasi wegretuschiert. Diesem Effekt behilflich ist zudem der "Schlaftrunk“ der personifizierten, offenbar dunklen und geheimnisvollen Wälder, der verursacht, dass die ganze „verheerte Welt“ nicht nur von der Dämmerung verhüllt, sondern auch dem Bewusstsein unzulänglich wird, in den nächtlichen Schlaf versinkt. Nur noch jener „Turm, den der Wächter verließ“, ist zu erblicken; ein Verweis auf die aus den Fugen geratene Zeit ohne wachsame Vernunft und

6 Nur Peter Horn (1969) geht eingehender auf den „Standort der Dichterin“, wie er in der ersten Strophe von „Mein Vogel“ skizziert wird, ein, und vergleicht das Gedicht, wie zwei Jahre vor ihm schon Wolfdietrich Rasch (RASCH 1967: 276), mit T. S.Eliots „The Waste Land“ von 1922. Horn interpretiert das eindringlich wiederholte „Was auch geschieht“ folgendermaßen: ,[...] wie schlimm diese Welt es auch treibt, was an Unheil und Verwirrung sich auch ereignen mag, der Dichter und sein Vogel dürfen ihren Platz nicht verlassen." (HORN 1969) Allgemein wird die „verheerte Welt“ als „Ausgangsort des Dichtungsprozesses“ und als eine „negative Realität" bei Mehthild Oberle erwähnt (OBERLE 1990: 46).

7 Dementsprechend wird die Auszeichnung durch den „armselige[n] Stern“, einen klaren Hinweis auf Jüdisches im Gedicht „Alle Tage“, nur dann verliehen, „,wenn nichts mehr geschieht“ (Zeile 10, BACHMANN 1982 I: 46).

8 Siehe die Stelle in ihrem Kriegstagebuch, die die Bombardierung Klagenfurts thematisiert, der sie allein, ohne ihre Familie, ausgesetzt war und bei der sie sich weigerte, in den Bunker zu gehen: „Aber ich habe keine Angst mehr, nur wenn die Bomben fallen ein körperliches Gefühl, etwas verkrampft sich in mir. Aber in meinem Kopf habe ich mein Testament gemacht. Vielleicht ist es sündhaft, einfach sitzen zu bleiben und in die Sonne zu schauen. Aber ich kann nicht mehr in den Bunker gehen, stundenlang wenn das Wasser an den Felswänden herunterrinnt und die Luft so schlecht wird, dass man halb ohnmächtig wird. [...] Der Gedanke, dort womöglich mit allen wie in einer Viehherde zugrundezugehen, ist mir schauerlich. Wenigstens im Garten. Wenigstens in der Sonne." (BACHMANN 2011: 12) Berühmt ist auch der Satz in ihrem Kriegstagebuch, wo sie sich über die Erwachsenen, am Krieg Beteiligten, folgendermaßen abschätzig äußert: „Die Erwachsenen, die Herren ,Erzieher', die uns umbringen lassen wollen." (BACHMANN 2011: 14) 
leitenden Verstand. Diese Leerstelle füllt als Abschluss und Krönung der ersten Strophe die dämmerungsaktive Eule (parallel zu Arends Albatros, der auch spannungsvoll erst am Ende der ersten Strophe erscheint), und zwar fokussiert auf ihre Augen, die anstatt des Wächters vom Turm aus die Lage überblicken, „ruhig und stet“ (mit Umsichtigkeit und Zuversicht), also im denkbar großen Kontrast zu der Verheerung des Anfangs der Strophe.

Die dreizeilige zweite Strophe wiederholt zwar die Anfangszauberformel „Was auch geschieht“, schreitet aber fort zu direkter Ansprache des Vogels mit den affirmativen Worten „Du weißt deine Zeit“ (Zeile 6); womit auf Celans Gedicht „Corona“ (v.a. auf Zeile 14: „es ist Zeit, daß man weiß!“) in dem Sinne angespielt zu werden scheint, ${ }^{9}$ dass die Eule als Symbol „der die Dunkelheit des Nichtwissens durchdringenden Weisheit“ (BECKER 1992: 75) den Augenblick ihres rechten Einschreitens kennt. Dieses Einschreiten besteht zuerst einmal darin, dass sie, wie das lyrische Subjekt in der Du-Ansprache schildert, ihren Schleier nimmt und dem lyrischen Ich „durch den Nebel“ (Zeile 8) zufliegt.

Die erste Zeile der dritten Strophe wiederholt die zauberhafte Anapher der ersten und zweiten Strophe nicht mehr, sondern berichtet von einer Tätigkeit, die die Eule und das lyrische Ich nun gemeinsam verrichten: „Wir äugen im Dunstkreis, den das Gelichter bewohnt.“ Hier bekommt der „Nebel“ der vorherigen Zeile einen Schub in Richtung negative Konnotation, denn ein Dunst kann neben der nebligen Luft auch stickige, von starkem Geruch und Dampf erfüllte Atmosphäre umfassen; und darüber hinaus ist das Kompositum „Dunstkreis“ im Sinne „Milieu“, „Wirkungsbereich“ leicht abwertend zu verzeichnen. Auch das Wort „Gelichter“ klingt negativ, obwohl es ursprünglich „zur selben Mutter Gehörende“ umriss und obwohl es in seiner Mitte etymologisch täuschend das positiv wahrgenommene Substantiv „Licht“ enthält. Es drückt aber „Abfälligkeit gegenüber einer Person oder mehreren Personen“ aus im Sinne „Gesindel“, und sein verächtlicher Gebrauch übersteigt also noch jenen vom Wort „Dunstkreis“.

Für Wolfdietrich Rasch meint der Ausdruck „die Wesen der ,verheerten Welt'“, (RASCH 1967: 278), allerdings ohne jeglichen Halt im Text des Gedichts. Ulrich Thiem sieht darin gerade das Gegenteil: „Auch das ,Gelichter“ gehört nicht zur ,verheerten Welt': Es ist wach, tätig und gefährlich, während sie schläft." (THIEM 1972: 33) Er fragt sich, ob und inwiefern es für die ,Verheerung' verantwortlich ist und worin die ,Verheerung“ besteht, findet aber keine Antwort und muss resigniert feststellen, dass „durch eine Vielzahl von Deutungsmöglichkeiten“ das Gedicht „zu einem Geflecht fluktuierender semantischer Beziehungen wird.“ (THIEM 1972: 33-34) Dementsprechend sieht Mechthild Oberle den Ausdruck „Gelichter“ gar im Zusammenhang mit der Jagdsprache, worin „Gelichter“ als Sammelbegriff für Krähen, Elstern und Eichelhäher dient (OBERLE 1990: 40-41), während Peter Horn in ihm rein abstrakt „das Gemeine, Wertlose“ (HORN 1969: Anm. 51) erblickt.

Eine Möglichkeit scheint die Forschung bisher nicht erwogen zu haben: Dass sich der Ausdruck „Gelichter“ sprach- und gedankenkritisch zu Martin Heidegger als dem

9 Bachmanns Antworten gerade auf dieses Gedicht aus der Sammlung Mohn und Gedächtnis (1952), ihr von Celan in Liebe zugeeignet, bestimmen, zusammen mit dem ihr ebenfalls gewidmeten Gedicht „Stille!“, wesentlich noch den Roman Malina von 1971. (WEIGEL/BÖSCHENSTEIN 2000:7) 
Kontrahenten von Ingeborg Bachmanns Dissertation beziehen könnte. Denn die Standardbegriffe dieses wesentlich in den Nationalsozialismus involvierten Philosophen (von 1933 bis zum Kriegsende Mitglied der NSDAP; vor allem während seines Rektorats 1933 bis 1934 stark an Propaganda und Gleichschaltungspolitik des NS-Staats beteiligt) umfassen zum Beispiel ähnlich gebildete Wörter wie „Geviert“ (für die Welt) oder „Gestell“ (für die Technik), weiterhin den Neologismus „lichten“; von Theodor W. Adorno, mit dem Bachmann befreundet war, 1964 pauschal als Jargon der Eigentlichkeit kritisiert. Auch das Verb „bewohnen“, im Hinblick auf das "Gelichter“ im Grunde zu ,hoch', könnte Heideggers pathetischen, der profanen Welt abholden, ethisch hohlen Sprachgebrauch attackieren, beispielsweise vor dem Hintergrund seines Hölderlinschen Aufsatzes „...dichterisch wohnet der Mensch..." von 1951, den Bachmann bestimmt kannte und der eskapistisch verstanden werden kann.

Das „Gelichter“ wäre demnach etwas Eigenes, Nahes (dem ursprünglichen Sinne „Geschwister“, „Menschen übereinstimmender Art, Sippe, Zunft“ entsprechend; ${ }^{10}$ zum Beispiel das eigene Volk), das aber negativ, täuschend, vulgär geworden ist und abgewehrt werden muss; durchs Äugen, vorsichtiges Hinblicken und Spähen in seinem „Dunstkreis“. Dann würde sich das „Äugen“ des lyrischen Ich und der Eule weniger auf „das erste Attribut der Eule, ihre wachsamen, klaren Augen" und somit auf die Charakterisierung des Vogels beziehen (OBERLE 1990: 41), als vielmehr auf den Erkenntnisgewinn durch diese Tätigkeit, die zum rechten Sehen verhelfen soll, zum Zustand „Mir sind die Augen aufgegangen." (BACHMANN 1982 IV: 275)

Wichtig ist hierbei, dass der Vogel und das lyrische Ich kooperieren, wie die Zeilen 10 und 11 beweisen: die Eule folgt dem Wink des lyrischen Ich, ,stößt hinaus/ und wirbel[t] Gefieder und Fell - “. Das Enjambement unterstreicht die Dynamik und Reichweite dieses rasanten, stolzen Tuns des Vogels, die Alliteration in Zeile 11 verstärkt zudem den Eindruck von der Gestik des Wirbelns, die auf das Balzritual hinweisen kann (dann wäre die Eule ein Männchen, und, darüber hinaus, wie schon der Falke im „Falkenlied“, das Symbol des Geliebten ${ }^{11}$ ), aber auch auf die Aufforderung zum Kampf. Jedenfalls ist dies herrlich anzusehen, und der Vogel ist sich seiner Macht, die in der Kraft seiner Bewegungen sowie in der Pracht seines Gefieders liegt, wohl bewusst. Dass diese Macht enorm, beinahe unendlich ist, betont der Gedankenstrich am Ende dieser Strophe - nur noch einmal wird er uns im Rahmen des Gedichts begegnen, und zwar ganz an seinem Ende, in der vorletzten Zeile, die vom Erfliegen der Warte aus Licht spricht.

Interpretieren wir das unbändige Präsentieren von „Gefieder und Fell“ des Vogels als den Verlobungsritus, als das Liebes-Vorspiel, so überrascht das in der vierten Strophe erfolgte, dankbare Bekenntnis des lyrischen Ich an die Adresse des Vogels wenig. Er wird voller Inbrunst und als Antwort auf sein Werben „Mein eisgrauer Schultergenoß, meine Waffe" genannt, und es wird unterstrichen, dass eine bestimmte, ,jene“, Feder von ihm die einzige Waffe des Ich darstelle sowie "Schleier und Feder" von ihm den einzigen Schmuck.

10 DUDEN 1989: 228.

11 Auf die Nähe der Eule zum „Jagdfalken“ macht auch RASCH 1967: 279 aufmerksam. 
Aber auch wenn wir in der Gestik des Wirbelns am Ende der dritten Strophe eine Kampfansage (offenbar an das „Gelichter“) sehen, wird in der vierten Strophe, die wie die zweite und dritte nur drei Zeilen zählt, die Dankbarkeitsbekundung des lyrischen Ich an die Eule, deren Besitz und Wertschätzung das Ich insgesamt viermal durch das Possessivpronomen „mein“ an den Tag legt, nachvollziehbar. Im Unterschied zu Erich Arendts Albatros, der seinem lyrischen Ich zuerst, mit dem gleichen Wort „Genoss“, „der weiten Fahrt Genoss“ (Zeile 6 der ersten Strophe) und dann, ebenfalls possessiv, „[m]einer Seele tapferster Genoss“ (Zeile 5 der fünften Strophe) ist, bewegt sich Bachmanns Eule vor allem in dieser vierten Strophe, aber auch darüber hinaus, in ausgesprochen martialischen Zusammenhängen. Das zeigt gleich die erste Zeile der Strophe mit der Ansprache „[m] ein eisgrauer Schultergenoß, meine Waffe“, wie auch die Tatsache, dass ihre Feder in der zweiten Zeile als des Ich „einzige Waffe“ figuriert, mit einem Ausrufezeichen versehen. „Schleier und Feder“ dürfen zudem, spartanisch genug, den einzigen Schmuck des lyrischen Subjekts ausmachen. Das ,Schöne’, in diesem „Schmuck“ verkörpert, geht eine Allianz mit dem Kämpferischen und dem Poetischen (Feder, Schleier) ein und weist auf die Gestalt der Athene als Kriegsgöttin, Schutzgöttin und Göttin der Weisheit hin, ${ }^{12}$ der die Eule symbolisch zugeordnet ist.

Hatten die ersten beiden Strophen des Gedichts die Konzessivsätze „Was auch geschieht“ an ihrem Anfang, so wird dies in der fünften Strophe zu einem ebenfalls konzessiven und anaphorischen Wenn-auch-Satz variiert, der die Zustände beschreibt, denen das lyrische Ich beim Schreibprozess, oder rein existentiell, ausgesetzt ist. Seine Haut fängt bei einem „Nadeltanz unterm Baum“13 Feuer, „der hüfthohe Strauch“ versucht es, seine „Locke züngelt“. Hans Höller interpretiert diese Strophe psychologisch und poetologisch in Hinsicht auf die „konzentrierte Anstrengung des schreibenden Ich, die dem Werk gilt“: „Die am nächsten liegenden Ansprüche des Lustprinzips müssen abgewehrt und unterdrückt werden, damit das Ich seine weiter gesteckten Ziele nicht preisgibt: die richtungslose Bewegung der unsublimierten Sinnlichkeit, als ,Nadeltanz unterm Baum', als Züngeln und Sich-Wiegen, muß vom Verstand auf ein Ziel gelenkt werden.“ (beides HÖLLER 1987: 48)

Doch genauso gut können diese Zeilen im Gegenteil als ein Lobgesang an die Macht des extrem, bis schmerzhaft wahrgenommenen Erotischen und Sinnlichen verstanden werden, wie er uns auch in Gedichten wie „Erklär mir, Liebe“ oder „An die Sonne“ aus der gleichen Sammlung Anrufung des Großen Bären (1956) affirmativ begegnet oder noch ausgeprägter zwei Jahre später im Hörspiel Der gute Gott von Manhattan. ${ }^{14}$ Dann wäre der „Sterne Schutt“, der dem lyrischen Ich am Ende der Strophe „doch genau auf das Haar“

12 Vgl. THIEM 1972: 31.

13 Nach Ulrich Thiem handelt es sich hier um ein Wortspiel, „das sich das Stilmittel des Austauschs zunutze macht: Statt ,Tanz unterm Nadelbaum' heißt es ,Nadeltanz unterm Baum ' - ein in der zeitgenössischen deutschen Lyrik häufiges Verfahren." (THIEM 1972: 38)

14 Schon in den Briefen an Felician der frühen Bachmann sieht Patricia Broser 2009 punktuell eine „explizite Überlagerung von Gewalt- und Liebesphantasien“ und konstatiert für das gesamte Werk der Dichterin, dass sie „Liebesakte oft in einem Schmerzkontext [darstelle], der einen katholischen Sozialisierungshintergrund durchblicken lässt.“ (BROSER 2009: 93) 
„stürzt“, ein Bote des Himmlischen, Astralen, der mit dem Körperlichen (Haut, Haar, Hüfte) des lyrischen Ich eine Verbindung eingeht ähnlich wie Himmel und Erde, das Männliche und das Weibliche in der Eichendorffschen „Mondnacht“.

Geradezu entgegengesetzt dieser romantischen, geheimnisvollen Nacht ist allerdings die Nacht, in der das lyrische Ich sich in der dritten Zeile der sechsten Strophe befindet, und möglicherweise "brennt" auch sie schon in der fünften Strophe an seiner Haut im Sinne höchster Gefahr, ${ }^{15}$ die außerdem im „Nadeltanz“ der ersten Zeile der fünften Strophe semantisch mitschwingt. Dem würde auch der „Rauch“ am Anfang der sechsten Strophe entsprechen, der das lyrische Ich „behelmt“, wieder unter Verwendung von Kriegsmetaphorik und wieder als eine Anleihe bei der Kriegsgöttin Athene, dieser allerdings im Unterschied zu Mars für die Gerechtigkeit kämpfenden Göttin, deren Attribut der Helm ist. ${ }^{16}$ Erst „vom Rauch behelmt“ wisse das Ich aber paradoxerweise „wieder [...], was geschieht“, womit ein Verweis auf die „verheerte Welt“ des Anfangs des Gedichts hergestellt wird, die ja durch die „Dämmrung“ verborgen und durch den magischen „Schlaftrunk“ der Wälder vergessen werden sollte.

Dem Titel von Celans erstem Gedichtband Mohn und Gedächtnis entsprechend, der allein schon im Bild des Mohns erstens auf Schlaf und schmerzlinderndes Vergessen (Schlafmohn als Opium), zweitens aber auf das notwendige Erinnern (Klatschmohn als Symbol für das Gedenken an gefallene Soldaten) hinweist, bringt nach der Phase des Schlafs und der Bewusstlosigkeit der „Rauch“, insbesondere in einer solchen unromantischen, traumatischen Nacht, die zweite und wichtige Phase mit sich, in der das lyrische Ich sich seiner Vergangenheit und Gegenwart stellt und erfährt, was passiert (ist): Der Krieg und die Shoah, deren Verbrennungsöfen den Rauch in solchen Mengen und stellvertretend für Ermordete (s. Celans „Todesfuge“ wie Sachs' „O die Schornsteine“) lieferten, dass das profane Wort „Rauch“, ähnlich wie etwa das Wort „Rampe“, weitläufig stigmatisiert wurde.

Also ist es nicht unlogisch, dass gerade dieser Rauch die Erweckung des lyrischen Ich zur Nüchternheit und zum Wissen darüber, „was geschieht," hervorruft und somit die ,Dimension Auschwitz frei legt, ${ }^{17}$ mitten in der Nacht, die zugleich als geschichtliche Nacht zu verstehen ist und über das Konditionale des Wenn-Satzes hinausreicht. Gerade in dieser Situation muss der Vogel aufgerufen werden, als „Beistand des Nachts“. Dank ihm kann das lyrische Subjekt „befeuert" werden „in der Nacht“, die dadurch der romantischen, verheißungsvollen Nacht wieder angeglichen wird, in der es zum ,Knistern“ der Inspiration kommt, typischerweise vor dem Hintergrund des „dunklen Bestand[s]“

15 Vergleichbar „höchster Angst und fliegender Eile“ von Bachmanns spätem Roman Malina (BACHMANN 1982 III: 12). Auf die Parallelen in der Figurenkonzeption von „Mein Vogel“ und dem Roman Malina weist Patricia Broser hin: BROSER 2009: 103-104.

16 HALL 1991: 275-276.

17 Dass man auch bei Ilse Aichingers berühmter Spiegelgeschichte von dieser Dimension sprechen kann, bewies Katharina Meiser 2017. Sie zitiert u.a. Ueli Jaussis Satz, wonach die Protagonistin im Spiegel erkennt, „was geschehen ist und nicht hätte geschehen dürfen," sowie Hans Höllers Meinung, dass das Schreiben über das Sterben nach 1945 immer verlange, „den industriell organisierten Mord mitzudenken, den wir mit dem Wort ,Auschwitz‘ verbinden." (beide Zitate MEISER 2017: 45) 
der opaken Wirklichkeit, die aber dazu beiträgt, dass das Ich „den Funken“ aus sich schlägt und sie erhellt.

In der letzten Strophe, auch einer konditionalen Wenn-Strophe wie der vorletzten Strophe, imaginiert das lyrische Ich gar, dass es „befeuert“ bleibe „wie ich bin/ und vom Feuer geliebt,“ also im Zustand (der ihm eigenen) heißen Liebe und der Inspiration längere Zeit verharrt, was dann zu der fabelhaften Folge hat, dass „das Harz aus den Stämmen tritt,/ auf die Wunden träufelt und warm/ die Erde verspinnt [...]." ${ }^{18}$ Der Erhellung der Wirklichkeit durch das dichterische Wort folgt ihre Heilung, der erkenntnisstiftenden Funktion von Literatur ihre therapeutische Aufgabe. Mitten in dieser Strophe finden wir jedoch, in Klammern verborgen, dadurch aber auch hervorgehoben, den Preis, den das lyrische Ich für seine welterhellende und weltrettende Mission zu zahlen bereit ist; und zwar im Zusammenhang mit „seinem“ Vogel, der Eule, die hier schließlich als Raubvogel stark exponiert wird: „(und wenn du mein Herz auch ausraubst des Nachts,/ mein Vogel auf Glauben und mein Vogel auf Treu!)“.

Ein vorletztes Mal begegnet uns hierbei der mutige, opferbereite Konzessivsatz des lyrischen Ich („und wenn du mein Herz auch ausraubst des Nachts“), diesmal, nach zweimaligem „Was auch geschieht“ (an der Spitze der ersten und zweiten Strophe) und nach einmaligem „Wenn auch“ der fünften Strophe, mit Bezug auf das eigene Herz, das Intimste, was es aufbieten kann. Zwei letzte Male wird zudem die Zugehörigkeit von lyrischem Ich und der Eule durch das Possessivpronomen „mein“ beschwören; im Zusammenhang mit emotionell so stark involvierenden Wörtern wie „Glauben“ und „Treu“ (in umgekehrter Reihenfolge bilden sie das Idiom „auf Treu und Glauben“, der auch rechtlich verankert ist und das Verhalten eines redlich und anständig handelnden Menschen umfasst) und mit dem zweiten, letzten Ausrufezeichen versehen, das dem in der Klammer Verborgenen den Charakter einer fast schmerzlichen Anflehung verleiht.

Nach all dem „rückt jene Warte ins Licht“ und schließt sich der Kreis, der in der ersten Strophe auf dem „Turm, den der Wächter verließ“ und in der „Dämmrung“ begonnen hatte. Während am Anfang des Gedichts es die Augen der Eule waren, die Ruhe, Kontinuität und Einsicht vermittelten (Zeile 5: „blicken ruhig und stet die Augen der Eule herab“), wird nun diese bestrahlte „Warte“ von der Eule „in herrlicher Ruhe“ erflogen, und die Eule, die in der Klammer noch unlängst als potentielle nächtliche Räuberin des Herzens des lyrischen Subjekts figurierte, als „besänftigt“ charakterisiert. Wieder erscheint hierbei, nach der Erwähnung der Herrlichkeit und der selbstsicheren, überlegten Ruhe des Fluges der Eule, ein Gedankenstrich; und wieder soll hier offenbar die Unendlichkeit der Kraft und Wirksamkeit des Vogels suggeriert werden. Sie sind es wohl, die auch aufgeboten werden müssen, um der „verheerte[n] Welt“ der ersten Zeile in ihrem ganzen Ausmaß zu trotzen.

Als Memento und Zuspruch, Zauberspruch in einem kann man dann die letzte Zeile des Gedichts verstehen, die aus einem Nebensatz ohne den dazugehörigen Hauptsatz besteht und durch die Anapher rhythmisch mit dem Beginn der ersten und zweiten

$18 \mathrm{Ob}$ die Baumstämme dadurch aber verbrannt werden, bleibt dahingestellt. Das Verb „spinnen“ sieht Patricia Broser darüber hinaus wieder im Zusammenhang mit der Göttin Athene, die den Menschen das Spinnen beibrachte. (BROSER 2009: 99) 
Strophe korrespondiert, sie auch als essentiell gewichtet. Ihre Inhalte werden durch das Zitat kurz einblendet, aber die fragmentarische Form lässt die eigentliche Spezifizierung dessen, was geschehen kann, geschehen ist oder geschieht, seltsam offen, im Positiven wie (und das vor allem) im Negativen potentiell unermesslich. Dadurch bleibt auch die Erkenntnis, die in der sechsten Strophe als ein wichtiger Gipfel des Gedichts figurierte (allerdings in einem konditionalen Zusammenhang: „Wenn ich vom Rauch behelmt/ wieder weiß, was geschieht“), eher nur paradigmatisch und symbolisch. Die Vorstellung vom Rauch der jüngsten Vergangenheit, d.h. der Shoah, der zum sichtbaren und tastbaren Helm verdichtet, einen Teil der Identität des lyrischen Ich fortan ausmacht, ist an die Existenz des Vogels gekoppelt, des „Beistand[s] des Nachts“, und an die Paradoxie, dass gerade das Dunkle und Undurchdringliche des „Bestand[s]“ schließlich den Funken der Inspiration und Erkenntnis stiftet. ${ }^{19}$

Doch das, „was auch geschieht“, kann auch diametral anderer Art sein, wenn wir es nicht als einen Konzessivsatz lesen, sondern als einen Subjektsatz in Bezug auf die Sequenz vor dem letzten Gedankenstrich des Gedichts. ${ }^{20}$ Denn dieser kann genauso gut auf die Existenz all dessen, was sich vor ihm in dieser Strophe befindet, hinweisen, und diese positive Existenz durch das betonte „auch“ („was [alles] auch geschieht“) bestätigen: die Wärme der heilsamen, heilbringenden Verspannung der Erde durch das Harz (das Wunden verschließt), wodurch die „verheerte Welt“ wieder intakt wird; die beleuchtete Warte, die der Vogel sanft und „in herrlicher Ruhe“ (trotz seiner Natur als Raubtier) erfliegt. Dadurch erfüllt der Gedankenstrich eine ähnliche Rolle wie jener in der Mitte des späten Gedichts „Enigma“ der Bachmann. Er fungiert einerseits als eine Ellipse, die das auslässt, was noch aufzuzählen, darzustellen wäre (und zwar, in unserem Gedicht, bis ins Unendliche hinaus), andererseits aber „als eine Ankündigung jenes anderen Modus des Sagens, der nach der Wendung des Gedankenstrichs aufgerufen wird." (VEDDER 2012: 348)

Dass beide Deutungsmöglichkeiten dieser Abschlusszeile, aber auch die diversen Deutungsmöglichkeiten von etlichen anderen Stellen des vorliegenden Gedichts, prinzipiell berechtigt sind, weist das Werk aus als ein typisches Gedicht der Moderne und als ein typisch hermetisches Gedicht zudem. ${ }^{21}$ In beiden Fällen jedoch, als ein Konzessiv- oder ein Subjektsatz, als ein Zauberspruch oder ein Zuspruch, vermag die Formel „was auch geschieht" den Rezipienten über all das hinweg zu trösten, was durch die geschichtlich konnotierte Nacht an Verheerendem auch stattfand, und eine Hoffnung auf Zukünftiges aussprechen. Diese Hoffnung gründet in der eigenartigen Koalition zwischen Dichter und seinem Vogel, der Inspiration, ${ }^{22}$ mit deren Hilfe die Gesetzlichkeiten des schöpferischen Prozesses nach der Katastrophe des Zweiten Weltkriegs und der Shoah umrissen

19 Dass das Paradoxe „in der Lyrik Ingeborg Bachmanns eine entscheidende, wenn nicht sogar die wichtigste, Komponente" darstelle, ist die zentrale These des Artikels von Zoltán Szendi (SZENDI 2004: 605).

20 Diese Lesart würde den kleinen Anfangsbuchstaben von „was auch geschieht“ rechtfertigen.

21 Thomas Sparr zitiert in diesem Zusammenhang Henri Pousseurs Begriff des „Möglichkeitsfeldes“, das den Rezipienten die Wahl überlässt, wie das betreffende Kunstwerk wahrzunehmen (zitiert in: SPARR 1989: 92). In Hinsicht auf die sog. hermetische Lyrik spricht man gewöhnlich vom „offenen Kunstwerk“.

22 Es ist nicht auszuschließen, dass Bachmann Préverts berühmte Paroles (1946) mit dem poetologischen 
werden; mit Phasen der Anästhesierung des Schmerzlichen bzw. des Traumatischen (Versenkung in die Konturlosigkeit der „Dämmrung“, Betäubung durch „Schlaftrunk“), seiner intellektuellen Untersuchung (,[̈̈]ugen im Dunstkreis“) und künstlerischen Bearbeitung (mithilfe „Schleier“ und insbesondere „jener Feder“), mit körperlichen Stigmata als Begleitumständen des Schöpferischen (5. Strophe) sowie mit (Re)artikulation der geschichtlichen Wunde und ihrer Sichtbarmachung durch „Funken“ (6. Strophe), schließlich mit ihrer Heilung (7. Strophe).

Dass das Gedicht nach all dem in eine verhaltene zwar, aber sich angesichts des Bösen durchaus zu behaupten wissende Utopie münden kann, ${ }^{23}$ beweist die Importanz, die der Dichtung in diesem Stadium des Bachmannschen Oeuvres zugeschrieben wird. Und dies durchaus nicht nur ,in eigener Sache', sondern vermutlich auch in Hinsicht auf Bachmanns Dichterkollegen, insbesondere Paul Celan, die es aufzurichten galt. Die Botschaft dabei ist klar: das wie auch immer aufgefasste „Gelichter“ ist bekämpfbar, das Gedicht besitzt eine gesellschaftliche Relevanz. Erst spätere Werke Bachmanns werden diese Annahme skeptisch überprüfen.

War das Bachmannsche Gedicht, wie wir gesehen haben, ein ziemlich komplexes Gebilde von oft ambivalenten und nicht endgültig dechiffrierbaren, obskuren Inhalten, Symbolen und Verweisen („Eule“ als Geliebter, Unterstützer und als bedrohender Raubvogel, „Nacht“ als geschichtliches Trauma und als Gebärmutter des Kreativen, „Feder“ als Schmuck und als Waffe, untereinander wetteifernde Bezüge zu der mythischen Pallas Athena), so scheint das Gedicht Erich Arendts erst einmal gar nicht hermetisch zu sein, sondern eher gut verständlich und, vor allem was das Metrum angeht, recht traditionell und regelmäßig. Schon seine zahlreichen, häufig das Schema ABABACC wiederholenden, vor allem Verben und Substantive einsetzenden Reime unterscheiden es von dem Gedicht Bachmanns, das, wie Patricia Broser bemerkt, über nur einen einzigen Reim (in Zeilen 8 und 14, und zwar reimt sich „mir“ auf „dir $)^{24}$ verfügt und damit der „Todesfuge“ Celans nahe kommt, deren einziger Reim in Zeilen 30 und 31 („sein Auge [des Meisters aus Deutschland] ist blau/ er trifft dich genau“) freilich um einiges bewegender ist.

Doch auch das Arendtsche Gedicht verfügt, trotz seiner quasi konformen Oberfläche, über eine eigentümliche Tiefe und Rätselhaftigkeit und verdient es, näher betrachtet zu werden; schon deshalb, weil es, parallel zu Bachmann, einige Merkmale des Schreibens nach den geschichtlichen Katastrophen der ersten Hälfte des 20. Jahrhunderts aufweist und darin mit Bachmanns „Eule“ einige Male frappierend korrespondiert. Wie bereits am Anfang des Kapitels erwähnt, zentrieren sich beide Gedichte um die Figur des Vogels, der eine Funktion als Vorbild und Kompagnon erfüllt und zudem als Stütze und Helfer dem lyrischen Ich dient. Offenbar ist es nach der Erfahrung des jüngst Vergangenen unumgänglich, sich der eigenen Identität mithilfe dieser Art von „Wappen im Gedicht“

Gedicht „Wie man einen Vogel malt“ kannte, das ebenfalls die künstlerische Kreativität und ihre Gesetzmäßigkeiten anhand der Figur eines Vogels schildert.

23 Deshalb wählte Patricia Broser 2009 u.a. das Gedicht „Mein Vogel“ zu ihrer Interpretation in Hinsicht auf Utopiekonzepte im Werk Ingeborg Bachmanns (so der Untertitel der Publikation; Ausführungen zum Gedicht Mein Vogel s. S. 89-105).

24 BROSER 2009: 99. 
zu vergewissern, um dadurch auch gegen die bei Bachmann wie bei Arendt als sehr unzulänglich empfundene gegenwärtige gesellschaftliche Situation ,gewappnet‘ zu sein.

Diese Ähnlichkeit wirkt bis in die Wahl der Lexik hinein. Während Arendt gleich in der ersten Strophe, und zwar, wie erwähnt, noch bevor er überhaupt seinen Vogel im Text des Gedichts vorstellt, ihn als „der weiten Fahrt Genoss[en]“ des lyrischen Ich bestimmt, spricht Bachmann am Anfang der vierten Strophe (also im Kern ihres Gedichts) von ihrem Vogel als dem „eisgraue[n] Schultergenoß[en]“ des lyrischen Subjekts, also von jemand, der im Kampf Schulter an Schulter neben dem Ich agieren soll. Dass gerade diese seine Funktion die heißersehnteste ist, beweist die ganze vierte Strophe des Bachmannschen Textes. Und parallel damit enthält die wärmste und ebenfalls ziemlich mittige, fünfte Strophe des Gedichts von Arendt in Zeile 33 die dankbaren Worte „Meiner Seele tapferster Genoss“, die dem Vogel und seiner Bedeutung für das lyrische Ich Arendts gelten. Die ganze Konstruktion dieser Strophe, die den charakteristischen, sehr dynamischen Fluss des Geschilderten durch eine Reihe von Adjektiven, syndetisch verbunden, weiter durch das oben erwähnte Fehlen einer Zeile sowie durch die Plazierung eines Doppelpunkts lähmt, unterstreicht das Bekenntnis an den Vogel, der die Seele des lyrischen Ich sinnvoll ergänzt, ähnlich, wie Bachmanns Eule Zutritt zum Herzen des lyrischen Ich hat. Nach Krieg und Katastrophe scheint es beiden Autoren ein Trost zu sein, in den Vögeln ein Wesen darzustellen, das körperlich wie geistig mobil ist sowie rasant, unbändig und unabhängig. Diese Eigenschaften werden gebraucht, um im Gedicht Widerstand gegen die unzureichende, weil unbewältigte jüngste Vergangenheit und ihre Schatten, die bis in die Gegenwart reichen, zu leisten.

Angesichts des Krieges ist, wie schon in der Barockpoesie und ihrer Antwort auf den Dreißigjährigen Krieg, auch noch eine andere Eigenschaft sehr von Bedarf: die Beständigkeit, in der Figur der Constantia versinnbildlicht. Bei Bachmann werden die Augen der Eule mit dieser Eigenschaft ausgestattet, sie blicken „ruhig und stet“, und stellen somit die Resistenz gegen das Chaos und die Destruktion der „verheerte[n] Welt“ dar, die bereits Andreas Gryphius zum Ausgangpunkt seines berühmten Gedichts „Tränen des Vaterlandes“ machte: „Wir sind doch nunmehr ganz, ja mehr denn ganz verheeret!“25 In diesem Gedicht konnte der Autor nur bitter beklagen, dass, so die Conclusio dieses Gedichts, „nun der Seelen-Schatz, so vielen abgezwungen“ wurde (GRYPHIUS 1988: 81), offenbar, weil es an einer Instanz mangelte, die „Beistand des Nachts“ und „Schultergenoss“ (Bachmann) oder der "Seele tapferster Genoss“ (Arendt) sein konnte. Darüber hinaus ist aber bei Arendt, neben dem hilfreichen, starken Vogel, noch eine andere Größe für die einigermaßen gute Wendung des Gedichts entscheidend: die Nacht. Sie ist es, die, wie in der sechsten Strophe (Zeile 41) ausgeführt, „doch stets den Albatros“ zu tragen fähig war und durch diese Beständigkeit für seinen Fortbestand, sein Leben sorgte. Doch eine solche Positivität liegt nur eine Zeile entfernt von genauem Gegenteil der hilfreichen, zuversichtlichen Nacht, denn der Vers 40 spricht von einer Nacht, die „die Sternensicht verschloß“, also düster und negativ war. Dadurch kommt es auf minimaler Fläche von zwei benachbarten Zeilen gerade dort zu einer Brechung des 
poetischen Bilds, wo auch Bachmann, in Vers 25 und 26 ihres Gedichts, eine Ambivalenz inszenierte: beim Nachtsymbol. Auch bei Arendt ist nämlich, und zwar ebenfalls im Rahmen eines einzigen Verspaars, die Nacht einerseits bedrohlich (weil sie „die Sternensicht verschloß“), und zugleich hilfreich (weil sie, trotz der Bedrohung, „doch stets den Albatros [trug]“). Wobei das Wort „doch“ die gegen alles Erwarten bewältigten Hindersnisse nahelegt und das Wort „stets“ eben die beruhigende zeitliche Kontinuität.

Dass die erste Arendtsche Gedichtsammlung schließlich Trug doch die Nacht den Albatros benannt wurde, d. h. ohne die Zeit- oder Umstandsbestimmung „stets“, die in Zeile 41 des Albatros-Gedichts eine so wichtige Funktion erfüllt („trug die Nacht doch stets den Albatros“ heißt es hier), kann aus Gründen des Schutzes vor einem zu langen Titel erfolgt sein, kann aber noch auf etwas anderes hindeuten. Möglicherweise reduzierte sich der positive Glaube auf Zukünftiges bei dem Dichter in der Zeit zwischen dem Verfassen des Kerns des Gedichts 1941 und der Veröffentlichung der Gedichtsammlung 1951, möglicherweise bekam sein Geschichtsoptimismus in dieser Zeit ernste Risse. Michaela Wolf und Georg Pichler zufolge war nämlich Arendts „Trauma der Rückkehr“ viel tiefer als sein eigentliches Trauma der Flucht, und das, obwohl seine Flucht oft äußerst lebensbedrohlich war: „Einen weitaus größeren Bruch als das Exil stellte in Arendts Biografie und in seinem Werk die Rückkehr nach Deutschland dar. [...] Weder erschloss sich ihm die deutsche Landschaft, noch behagte ihm, trotz seiner privilegierten Stellung als Exilant, das Leben in der DDR.“ (WOLF/PICHLER 2007: 22) Bei Stefan Wieczorek erfahren wir triftige Gründe für das Letztere: „Als Erich Arendt in die DDR kommt, muß er feststellen, daß Weggefährten, die parteipolitisch aktiver waren als er und denen er viel zu verdanken hat, allmählich Opfer von Schauprozessen werden." (WIECZOREK 2001: 44) Schon bevor Arendt überhaupt die DDR erreicht, wird er mit Vorbereitungen des stalinistischen Slánký-Prozesses in Prag konfrontiert, der zwei seiner sehr nahen Freunde (schon aus der Zeit des spanischen Bürgerkriegs) ernsthaft bedroht: Artur London und Noël Field. Rückblickend misst er dieser Desillusionierung große Bedeutung zu:

\begin{abstract}
„Ein großer Schnitt, ein Messerschnitt durch meinen ideellen Traum, geschah mit den Prager Prozessen. Da tauchte der, den ich sehr gut kannte und als treuen Genossen erlebt hatte, Noël Field ständig auf. Und ich wußte bevor Chruschtschow gesprochen hat bereits wieviel Lüge, wieviel Verbrechen in diesen Prager Prozessen waren. [...] Ich wußte, daß Artur London, der einer der saubersten, ganz sich einsetzenden Kommunisten gewesen ist, der den Rückzug der Brigaden als letzter in Spanien gedeckt hat im Schnee, mit einem Maschinengewehr, daß das kein Verräter war, sondern daß der Prozeß in Prag der Verrat war an der Idee des Sozialismus. Und diese Dinge, die in anderen Ländern parallel liefen, haben mich von der Realität des Landes, in dem ich nun lebte, entfernt.“ (ARENDT zitiert in WIECZOREK 2001: 46)
\end{abstract}

Die Ballade vom Albatros, wie das Gedicht in der Sekundärliteratur immer wieder bezeichnet wird, soll wohl auch angesichts dieser Vorkommnisse allegorisch die Behauptung des lyrischen Subjekts mitten in "Not und Knechtschaft“ darstellen, und zwar dadurch, dass „wilder Ruf“ des Vogels vom lyrischen Ich adaptiert wird und zu seiner eigenen Artikulation des Protestes gegen das Negative umgewandelt wird. 
Doch diese Leistung geschieht, wie der Anfang der letzten Strophe einräumt, „[n]ur in Träumen noch“ und erst „nach Jahren“, was dem Ganzen eine wehmütige elegische Färbung gibt. Schon Suzanne Shipley Toliver hat darauf hingewiesen, dass es Arendt in diesem Gedicht darum gehe, seine persönlichsten Kommentare in die Allegorie zu hüllen (vgl. TOLIVER 1984: 69); offenbar aus Angst, sie offen darzustellen und sich dadurch preiszugeben. In dieser Hinsicht würde das Gedicht eine erste Stufe auf dem Weg zu späteren völlig hermetischen Gedichten markieren und Wunbergs Motto zur Begründung des Hermetischen erfüllen: „Was niemand versteht, ist auch durch niemanden gefährdet" (WUNBERG 1989: 247). Demnach wäre das Albatros-Gedicht Arendts eher als ein existentielles Opus des Autors vor dem Hintergrund des Wahnsinn des Jahrhunderts (Hans Dieter Zimmermann, 1992) zu verstehen und seine rhythmische Regelmäßigkeit nicht balladesk motiviert, sondern eher als Mimikry zur Beförderung aufrührerischer Inhalte von der Macht des Zorns (diese Vokabel begegnet uns viermal im Text des Gedichts und soll bestimmt im Gedächtnis des Lesers als eine zentrale haften bleiben). Auch seine äußere Konventionalität sollte offenbar täuschen und möglicherweise subversiv wirken.

Nicht zuletzt kann das Gedicht Arendts auch als eine pointierte poetologische Aussage verstanden werden und darin eine weitere Parallelität zu Bachmanns „Mein Vogel“ aufweisen. Es soll uns lehren, vor allem anhand des unbändigen, im höchsten Maß freien Verhaltens des Vogels im Sturm, welches insbesondere die fünfte Strophe (die einzige sechszeilige des Gedichts) in pathetischen Worten schildert, das „Herz des Sturmes“ (Zeile 29) wahrzunehmen und „von Empörung heiß, von Zorn lebendig“ (Zeile 30) zu fühlen wagen, vor allem aber: den Zorn der eigenen Seele zu artikulieren, zu schreien: „Meiner Seele tapferster Genoss,/ rief er ihren Zorn, der Albatros“ (Zeile 33-34). Sogar noch die vorletzte Zeile des Gedichts (Zeile 54) spricht vom „[w]ilde[n] Ruf, der durch den Himmel schoß" als vom Vermächtnis des Vogels an das lyrische Ich. Auch Arendt glaubt, parallel zu Bachmann in ihrem Eule-Gedicht, in diesem Augenblick noch ziemlich ungebrochen an die Möglichkeit der gesellschaftlichen Wirkungskraft von Literatur, von Gedicht; auch Arendt wird sie aber bald skeptisch hinterfragen müssen.

Fest aber steht, dass beide Autoren angesichts der Katastrophe ihrer jüngsten Vergangenheit, durch den Zweiten Weltkrieg und die Shoah, aber auch die gewaltsamen Ereignisse davor und danach repräsentiert, die Kräfte mobilisieren und dass sie eine Poetologie entwickeln wollen, die diesen Herausforderungen gerecht wäre - was natürlich schon in Anbetracht der Verheerungen keine einfache Aufgabe ist. Sie fühlen offenbar, dass ihre jeweiligen lyrischen Ich allein zu schwach sind und dass sie eine symbolische Unterstützung und Überhöhung brauchen von einer Instanz, die stärker und stabiler ist als sie; sei es der Vogel Albatros und der Vogel Eule, sei es Odysseus oder Pallas Athena. Dank ihnen können sie sich, im Unterschied etwa zu Andreas Gryphius und seinen in Reaktion auf den Dreißigjährigen Krieg entstandenen „Tränen des Vaterlandes“, die ebenfalls von Verheerungen als Folge der kriegerischen Auseinandersetzungen ausgehen, einigermaßen behaupten. Arendts Albatros besticht dabei durch deklamatorisch wirksam eingesetzte Bilder des Dynamischen (Sturm und Zorn als Schlüsselwörter), Bachmanns Eule dagegen eher durch ihre Ruhe und die magische, dreimal wiederholte 
Beschwörungsformel „W/was auch geschieht“ im Zusammenhang mit ihr; was in etwa einem genderspezifischen Rollenverständnis entspricht.

Beide Gedichte sind zudem als Antwort auf den Krieg bzw. die Shoah mehrdeutig und verschiedenen, auch sich ausschließenden Interpretationsansätzen offen. Dies ist eines der typischen Merkmale des Hermetischen und in Bachmanns Gedicht bestimmt stärker aufzufinden als bei Arendt, dessen Allegorien jedoch den Interpreten auch vor schwierige Entscheidungen stellen und sehr Rätselhaftes entfalten; als ob sich in ihnen der Autor schützen würde vor Preisgabe des eigenen Ich und seiner Bedürfnisse (auch dies charakteristisch für hermetische Dichter). Viel eher als eine Ballade wäre demnach sein Gedicht als ein existentielles einzuschätzen, und auch das Bachmannsche Gedicht verdient u.a. bestimmt diese Bezeichnung. Denn Arendt wie Bachmann reflektieren die Komplexität ihrer Zeit, indem sie Paradoxien darstellen, was zu Symbolbrechungen führt (die Nacht als Verheißung und Gefährdung, der Vogel als Stütze und Gefahr).

Als poetologische Gedichte dokumentieren die Texte bei beiden Autoren eine Etappe in ihrer dichterischen Entwicklung, die noch hoffnungsvoll ist und der noch kreative Energien der Erneuerung nach Krieg und Katastrophe eigen sind; alsbald sollte dem jedoch eine Verdüsterung und Aufgabe des Glaubens an die Macht des Wortes folgen. Obwohl beide Texte zugleich das artikulieren, was unabhängig von Zeit und der jeweiligen Gesellschaft ist, die Mühen und Gesetzmäßigkeiten des dichterischen Schaffens, bleiben sie trotzdem der „Not und Knechtschaft“ (Arendt), der „verheerte[n] Welt“ (Bachmann) als ihrem Ausgangspunkt verbunden: als ein Mahnmal und als Beherzigung an die Zeitgenossen gedacht.

\section{Literaturverzeichnis}

\section{Primärliteratur:}

ARENDT, Erich (2003): Kritische Werkausgabe. Hrsg. von Manfred Schlösser. Bd. I: Gedichte 1925-1959. Berlin.

BACHMANN, Ingeborg (2011): Kriegstagebuch. Mit Briefen von Jack Hamesh an Ingeborg Bachmann. Berlin.

BACHMANN, Ingeborg (1982): Werke. Erster Band: Gedichte, Hörspiele, Libretti, Übersetzungen. München - Zürich.

BACHMANN, Ingeborg (1982): Werke. Dritter Band: Todesarten: Malina und unvollendete Romane. München - Zürich.

BACHMANN, Ingeborg (1982): Werke. Vierter Band: Essays, Reden, Vermischte Schriften, Anhang. München - Zürich.

BACHMANN, Ingeborg (1983): Wir müssen wahre Sätze finden. Gespräche und Interviews. München.

BAUDELAIRE, Charles (1979): Albatros. In: ders.: Víno samotářovo. Praha, S. 31.

CELAN, Paul (1968): Ausgewählte Gedichte. Zwei Reden. Frankfurt am Main.

GRYPHIUS, Andreas (1988): Tränen des Vaterlandes, Anno 1636. In: Stephan Hermlin (Hrsg.): Deutsches Lesebuch. Leipzig, S. 80-81. 


\section{Sekundärliteratur:}

BECKER, Udo (1982): Lexikon der Symbole. Freiburg - Basel - Wien.

BROSER, Patricia (2009): Ein Tag wird kommen... Utopiekonzepte im Werk Ingeborg Bachmanns. Wien.

DUDEN (1989): Etymologie. Herkunftswörterbuch der deutschen Sprache. Bd. 7. Mannheim Wien - Zürich.

EMMERICH, Wolfgang (1984): Mit rebellischem Auge. Die Exillyrik Erich Arendts. In: Text + Kritik, Heft 82/83: Erich Arendt, S. 27-44.

HALL, James (1991): Slovník námětů a symbolů ve výtvarném umění. Praha.

HEIDEGGER, Martin (1978): „...dichterisch wohnet der Mensch...“ (1951). In: ders.: Gesamtausgabe, I. Abteilung: Veröffentlichte Schriften 1910-1976. Frankfurt am Main, S. 181-197.

HÖLLER, Hans (2001): Ingeborg Bachmann. Reinbek bei Hamburg.

HÖLLER, Hans (2008): Schreiben gegen den Krieg. Ein literaturgeschichtlicher Essay zur Ausstellung. In: HÖLLER, Hans/PÖCHEIM, Helga/SOLIBAKKE, Karl Ivan (Hrsg.): Ingeborg Bachmann. Schreiben gegen den Krieg. Eine Ausstellung. Wien.

HORN, Peter (1969): Anruf und Schweigen in den Gedichten von Ingeborg Bachmann. In: Acta Germanica 4/1969, S. 67-103. http://www.academia.edu/4487595/_Anruf_und_Schweigen_ in_den_Gedichten_von_Ingeborg_Bachmann._In_Acta_Germanica._S.67-103 (21.2.2018).

MEISER, Katharina (2017): Die „Dimension Auschwitz“ in Ilse Aichingers Spiegelgeschichte. In: Weimarer Beiträge 1/2017, 63. Jahrgang, S. 44-58.

OBERLE, Mechthild (1990): Liebe als Sprache und Sprache als Liebe. Die sprachutopische Poetologie der Liebeslyrik Ingeborg Bachmanns. Frankfurt am Main - Bern - New York - Paris.

RASCH, Wolfdietrich (1967): Drei Interpretationen moderner Lyrik. I. Ingeborg Bachmann: „Mein Vogel“. In: ders.: Zur deutschen Literatur seit der Jahrhundertwende. Gesammelte Aufsätze. Stuttgart.

SPARR, Thomas (1989): Celans Poetik des hermetischen Gedichts. Heidelberg.

SZENDI, Zoltán (2004): Das Paradoxe der Ich-Befindlichkeit in der Lyrik Ingeborg Bachmanns. In: Zeitschrift für Deutsche Philologie, Bd. 123, H. 4, S. 605-617.

THIEM, Ulrich (1972): Die Bildsprache der Lyrik Ingeborg Bachmanns. Köln.

TOLIVER, Suzanne Shipley (1984): Exile and the Elemental in the Poetry of Erich Arendt. New York - Berne - Frankfort on the Main - Nancy.

ULLRICH, Helmut (1969): Es trug die Nacht den Albatros. Die Welterfahrung des Lyrikers Erich Arendt im Spiegel seiner Dichtungen. Variationen zur Thematik bewegter und bewegender Zeiten. In: Neue Zeit, 21.5.1969. http://www.planetlyrik.de/erich-arendt-aus-funf-jahrzehnten/2012/09 (21.2.2018).

VEDDER, Ulrike (2012): „Verhoffen“: Gedankenstriche in der Lyrik von Ingeborg Bachmann, Nelly Sachs und Paul Celan. In: NEBRIG, Alexander/SPOERHASE, Carlos (Hrsg.): Die Poesie der Zeichensetzung. Studien zur Stilistik der Interpunktion. Berlin [u.a.], S. 345-361.

WEIGEL, Sigrid/BÖSCHENSTEIN, Bernhard (2000): Paul Celan - Ingeborg Bachmann. Zur Rekonstruktion einer Konstellation. In: BÖSCHENSTEIN, Bernhard/WEIGEL, Sigrid (Hrsg.): Ingeborg Bachmann und Paul Celan. Poetische Korrespondenzen. Frankfurt am Main.

WIECZOREK, Stefan (2001): Erich Arendt und Peter Huchel. Kleine Duographie sowie vergleichende Lektüren der lyrischen Werke. Marburg.

WOLF, Michaela/PICHLER, Georg (2007): Übersetzte Fremdheit und Exil - Grenzgänge eines hybriden Subjekts. Das Beispiel Erich Arendts. In: Exilforschung 25/2007: Übersetzung als transkultureller Prozess. München, S. 7-29. 
WUNBERG, Gotthart (1989): Hermetik - Änigmatik - Aphasie. Zur Lyrik der Moderne. In: Poetik und Geschichte. Tübingen, S. 241-249.

ZIMMERMANN, Hans Dieter (1992): Der Wahnsinn des Jahrhunderts. Die Verantwortung der Schriftsteller in der Politik. Stuttgart.

Mgr. Jana Hrdličková, Ph.D. / jhrdlickova@yahoo.de

Univerzita J. E. Purkyně v Ústí nad Labem,

Filozofická fakulta, Katedra germanistiky,

Pasteurova 3571/13, 40096 Ústí nad Labem, CZ 
